\title{
Coxiella burnetii and Leishmania mexicana residing within similar parasitophorous vacuoles elicit disparate host responses
}

\author{
Jess A. Millar ${ }^{1}$, Raquel Valdés ${ }^{2}$, Fenil R. Kacharia ${ }^{1}$, Scott M. Landfear ${ }^{2}$, \\ Eric D. Cambronne ${ }^{2}$ and Rahul Raghavan ${ }^{1 *}$
}

'Department of Biology and Center for Life in Extreme Environments, Portland State University, Portland, OR, USA,

${ }^{2}$ Department of Molecular Microbiology and Immunology, Oregon Health and Science University, Portland, OR, USA

\section{OPEN ACCESS}

Edited by:

Gregoire S. Lauvau, Albert Einstein College of Medicine,

USA

Reviewed by:

Lynn Soong,

The University of Texas Medical Branch at Galveston, USA

Emilio Luis Malchiodi,

University of Buenos Aires, Argentina

*Correspondence:

Rahul Raghavan

Department of Biology and Center for Life in Extreme Environments, Portland State University, Portland,

OR 97201, USA

rahul.raghavan@pdx.edu

Specialty section:

This article was submitted to

Microbial Immunology,

a section of the journal

Frontiers in Microbiology

Received: 19 May 2015

Accepted: 21 July 2015

Published: 07 August 2015

Citation:

Millar JA, Valdés R, Kacharia FR,

Landfear SM, Cambronne ED and Raghavan $R$ (2015) Coxiella

burnetii and Leishmania mexicana residing within similar parasitophorous vacuoles elicit disparate host responses.

Front. Microbiol. 6:794. doi: 10.3389/fmicb.2015.00794
Coxiella burnetii is a bacterium that thrives in an acidic parasitophorous vacuole (PV) derived from lysosomes. Leishmania mexicana, a eukaryote, has also independently evolved to live in a morphologically similar PV. As Coxiella and Leishmania are highly divergent organisms that cause different diseases, we reasoned that their respective infections would likely elicit distinct host responses despite producing phenotypically similar parasite-containing vacuoles. The objective of this study was to investigate, at the molecular level, the macrophage response to each pathogen. Infection of THP-1 (human monocyte/macrophage) cells with Coxiella and Leishmania elicited disparate host responses. At 5 days post-infection, when compared to uninfected cells, 1057 genes were differentially expressed (746 genes up-regulated and 311 genes downregulated) in C. burnetii infected cells, whereas 698 genes (534 genes up-regulated and 164 genes down-regulated) were differentially expressed in L. mexicana infected cells. Interestingly, of the 1755 differentially expressed genes identified in this study, only 126 genes $(\sim 7 \%)$ are common to both infections. We also discovered that 1090 genes produced mRNA isoforms at significantly different levels under the two infection conditions, suggesting that alternate proteins encoded by the same gene might have important roles in host response to each infection. Additionally, we detected 257 micro RNAs (miRNAs) that were expressed in THP-1 cells, and identified miRNAs that were specifically expressed during Coxiella or Leishmania infections. Collectively, this study identified host mRNAs and miRNAs that were influenced by Coxiella and/or Leishmania infections, and our data indicate that although their PVs are morphologically similar, Coxiella and Leishmania have evolved different strategies that perturb distinct host processes to create and thrive within their respective intracellular niches.

Keywords: Coxiella burnetii, Leishmania mexicana, parasitophorous vacuole, isoform, miRNA

\section{Introduction}

Macrophages that phagocytize pathogens and recruit other immune cells are critical for the elimination of potential infections. Within macrophages, engulfed pathogens are transported inside phagosomes that later fuse with lysosomes to generate the phagolysosome. Most pathogens are degraded within the phagolysosome, which has a very harsh environment (low $\mathrm{pH}$, 
high concentration of lysosomal hydrolases, presence of cationic peptides etc.; Kinchen and Ravichandran, 2008; Flannagan et al., 2009). Several pathogens have evolved strategies to survive and replicate within macrophages: Toxoplasma gondii prevents the fusion of its vacuoles with the endosomal pathway; Salmonella enterica Typhimurium, Mycobacterium tuberculosis, and Legionella pneumophila block maturation of phagosomes into phagolysosomes; Shigella flexneri and Listeria monocytogenes escape into cytoplasm from phagosomes before lysosomal fusion; Trypanosoma cruzi escapes from phagosomes after fusion with lysosomes (Swanson and Fernandez-Moreia, 2002; Flannagan et al., 2009).

Unlike most other pathogens, Coxiella (a bacterium) and Leishmania (an eukaryote) have independently evolved the ability to thrive in a parasitophorous vacuole (PV) that is derived from the fusion of phagosomes with lysosomes (Voth and Heinzen, 2007; Alix et al., 2011). Coxiella burnetii (the only defined species within this genus) causes human $\mathrm{Q}$ fever and chronic endocarditis. The bacterium is shed in milk, urine, and birth products of animals, and can survive in the environment via a "spore-like" form called the small cell variant (SCV). C. burnetii is usually acquired via inhalation, and initially infects alveolar macrophages but then spreads to mononuclear phagocytes of other tissues. Within the macrophage, SCV transforms into a metabolically active form called the large cell variant (LCV), and multiple Coxiella-containing vacuoles merge to form a single large vacuole that fuses with endolysosomal vesicles to give rise to the mature Coxiella PV (van Schaik et al., 2013).

Leishmania is a genus of trypanosomatid parasite that comprises several species of medical and veterinary importance that cause cutaneous, mucocutenous, or visceral diseases. It has a dimorphic lifecycle that alternates between an extracellular promastigote form in insect vectors and an intracellular amastigote from in mammalian hosts (Herwaldt, 1999). The primary host cells of Leishmania are macrophages, but it can also infect neutrophils, fibroblasts, and dendritic cells (Contreras et al., 2014). Similar to the biogenesis of Coxiella PV, the Leishmania-containing vacuole also fuses with endolysosomal vesicles to give rise to the mature Leishmania PV. However, the morphology of PV varies among different Leishmania species. In several species, including $L$. donovani, $L$. infantum, and L. major, only one or two amastigotes reside within each PV, which segregates into new vacuoles after parasite replication. In contrast, as observed for Coxiella PVs, parasites of the Leishmania mexicana complex such as L. mexicana and L. amazonensis form communal PVs that continuously enlarge as the parasites replicate (Real et al., 2010). Interestingly, coinfection studies have shown that PVs formed by L. amazonensis amastigotes can fuse with $C$. burnetii PVs but not with PVs containing L. major amastigotes, suggesting that the intracellular niches generated by L. mexicana complex parasites and Coxiella may be compositionally rather similar (Veras et al., 1995; Rabinovitch and Veras, 1996; Real et al., 2010; Beare et al., 2011; Newton and Roy, 2011).

Both Coxiella and Leishmania actively participate in the creation of their respective PVs, which are intracellular compartments distinct from canonical phagolysosomes. To begin to understand how the two distantly related pathogens generate phenotypically similar PVs, we compared host gene expression in human macrophage cells (THP-1) infected with either C. burnetii or L. mexicana. Our data show that the bacterium and the eukaryote elicit distinct host messenger RNA (mRNA) and microRNA (miRNA) responses, indicating that despite their superficial similarity, generation, and maintenance of the Coxiella PV and Leishmania PV involve distinct host processes.

\section{Materials and Methods}

\section{C. burnetii and L. mexicana Infection of THP-1 cells, RNA Extraction, and RNA-seq}

THP-1 cells (TIB-202; ATCC) were maintained in RPMI 1640 medium (Gibco) supplemented with $10 \%$ fetal calf serum (Gibco) at $37^{\circ} \mathrm{C}$ in $5 \% \mathrm{CO}_{2}$. Cells were incubated in the presence of $200 \mathrm{nM}$ phorbol 12-myristate 13-acetate (PMA; EMD Biosciences) for $24 \mathrm{~h}$ to induce differentiation into adherent, macrophage-like cells. Prior to infection, PMAcontaining medium was replaced with fresh RPMI without PMA. Cells were infected with either C. burnetii (Nine Mile phase II, RSA 493) or promastigotes of L. mexicana (MNYZ/BZ/62/M379) at an approximate multiplicity of infection of 25 and incubated for 5 days. Growth medium was replaced every two days and formation of Coxiella and Leishmania PVs was monitored microscopically. At 5 days post-infection, growth medium was replaced with $1 \mathrm{ml}$ of TRI reagent (Life Technologies) and total RNA was extracted, and genomic DNA was removed by DNase (Life Technologies) treatment, as per instructions. RNA from two samples each of uninfected, Coxiella-infected, and Leishmaniainfected THP-1 cells were used to prepare mRNA and small RNA Illumina sequencing libraries. To analyze gene expression, the six mRNA libraries were pooled into a single lane of an Illumina HiSeq $2000(2 \times 75$ cycles $)$. For miRNA identification, the six small RNA libraries were pooled into a single Illumina Miseq lane $(1 \times 50$ cycles $)$. All RNA-seq reads are available at National Center for Biotechnology Information Sequence Read Archive (Accession SRP045986).

\section{Mapping Sequencing Reads and Identification of Differentially Expressed Genes}

Reads were cleaned by removing adapters and were filtered by quality ( $>$ Q20) and length ( $>50 \mathrm{bp})$ using Trimmomatic v0.30 (Bolger et al., 2014). Homo sapiens reads were filtered for possible contamination by mapping to C. burnetii genome (NC_002971.3) using BWA MEM v0.7.5 (Li and Durbin, 2010) and $L$. mexicana genome (NZ_CADB00000000.1) using Tophat v2.0.11 (Kim et al., 2013). Final clean reads were mapped to H. sapiens Genome Reference Consortium Human Build 37 (GCF_000001405.13) using CLC Genomic Workbench v6.5. To identify differential gene expression, replicate data were pooled for pairwise comparisons and quantile normalized using CLC Genomic Workbench v6.5. Genes were filtered based on at least 10 raw reads mapping to each sample, and a $\log 2$ transformed fold change of one SD above or below the mean. Differentially expressed genes were chosen based on significance $(P<0.05$, 
FDR-corrected beta-binomial distribution test). Raw read counts mapped to each mRNA isoform were exported from CLC into EBSeq (Leng et al., 2013) and differential expression of isoforms was determined based on significant EBSeq values $(P<0.05$, FDR-corrected).

For quantitative PCR (qPCR) validation of gene expression, $1 \mu \mathrm{g}$ of DNase-treated RNA, and oligo-dT primers were used to prepare cDNA (Thermo Scientific). A subset of genes involved in host cell death (TGFB2, RIPK2, CYR61, CYP1B1, NFKBIA) was selected and qPCR was performed using SYBR green on an Agilent Mx3000P System. Fold difference value for each gene was calculated using the $2^{-\Delta \Delta C T}$ method with GAPDH as the control. As shown previously (Raghavan et al., 2012), to assess the correlation between expression estimates from RNA-seq and qPCR, we calculated the Pearson correlation coefficient between fold difference values calculated by each method for Coxiella and Leishmania-infected cells.

\section{Gene Ontology (GO) Analysis and Protein-Protein Interaction Networks}

GO terms were found using Database for Annotation, Visualization and Integrated Discovery (DAVID), and the GO FAT filter. GO-term enrichment tests were also performed with DAVID (Huang et al., 2009a,b). Kyoto Encyclopedia of Genes and Genomes (KEGG) pathways over-represented among differentially expressed genes were chosen based on the level of statistical significance $(P<0.01)$. Protein-protein interaction networks were visualized using STRING 9.1 (Franceschini et al., 2013). Proteins unconnected to the main graph were removed. Markov Clustering was performed on STRING confidence scores using an inflation factor of two to visualize subgraphs of interacting protein processes (Brohée and van Helden, 2006). GO-terms were overlaid onto the graphs using STRING to identify what processes were represented in the separate subgraphs.

\section{Identification of miRNAs}

Sequencing reads were cleaned by removing adapters and filtered by quality ( $>$ Q20) and length ( $>15$ bp) using Trimmomatic (Bolger et al., 2014). Replicate data was pooled and miRNAs were identified using CLC based on having an average of at least 10 reads mapped to mature $5^{\prime}$ or $3^{\prime}$ miRNAs annotated in mirBase (Kozomara and Griffiths-Jones, 2014).

\section{Results and Discussion}

\section{C. burnetii and L. mexicana Infections Induce Robust but Non-Overlapping Host Responses}

Human monocyte/macrophage cell line THP-1 was used to evaluate host responses against $C$. burnetii and L. mexicana. Previous studies have investigated host responses during early stages (6-72 hpi) of infections by C. burnetii and by various Leishmania species (Ren et al., 2003; Mahapatra et al., 2010; De Muylder et al., 2011; Rabhi et al., 2012, 2013); however, because the transformation from the infective form (SCV and promastigote, respectively) to the replicative form (LCV and
A

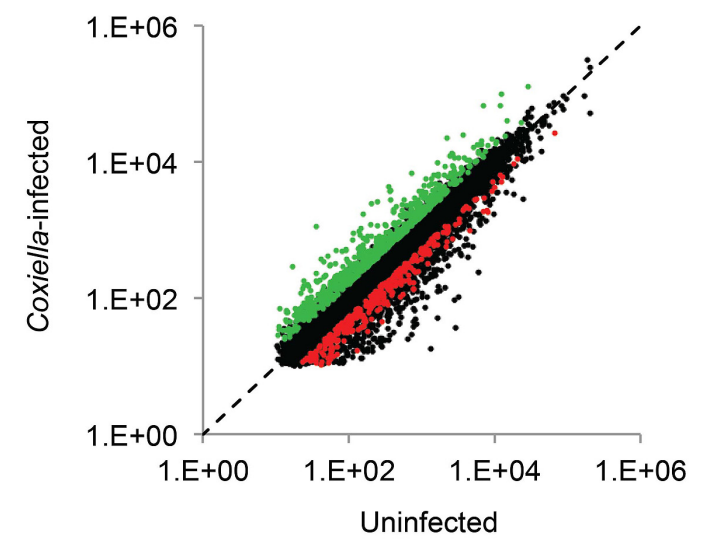

B

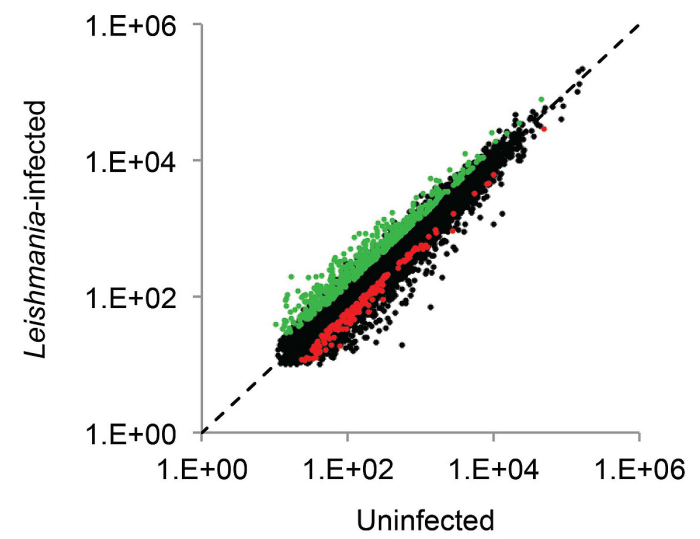

C

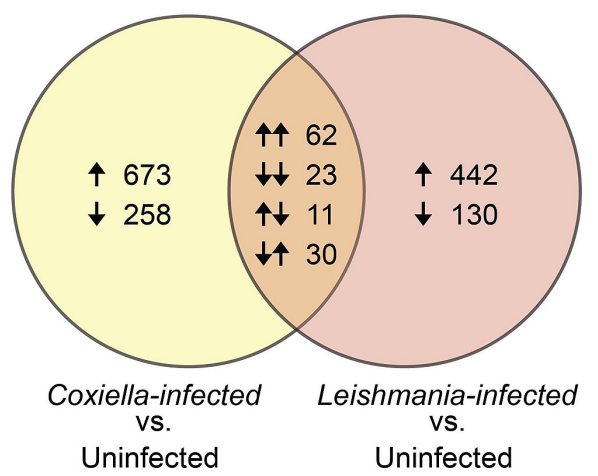

FIGURE 1 | Identification of differentially expressed genes. Gene expression in (A) Coxiella burnetii-infected and (B) Leishmania mexicana-infected THP-1 cells in comparison to uninfected THP-1 cells are shown. Differentially expressed genes are highlighted in red and green. (C) Comparison of genes differentially expressed in C. burnetii-infected and L. mexicana-infected cells. Arrows indicate up-regulation or down-regulation of genes.

amastigote, respectively) occur at differing rates in the two pathogens, we analyzed a later point during infection (5 days pi) when both pathogens have generated large PVs that fill most of the host cell volume. When compared to uninfected THP-1 cells, 1057 genes (746 up-regulated and 311 down- 
TABLE 1 | KEGG pathways enriched in Coxiella-infected and Leishmania-infected THP-1 cells.

\begin{tabular}{|c|c|c|c|c|c|}
\hline Sample & $\begin{array}{l}\text { KEGG } \\
\text { term }\end{array}$ & Description & Genes & $\begin{array}{l}\text { Fold } \\
\text { enrichment }\end{array}$ & $P$-value \\
\hline \multirow[t]{7}{*}{ Coxiella-infected } & hsa04210 & Apoptosis & $\begin{array}{l}\text { BID, IRAK2, TNF, XIAP, RELA, TP53, NFKBIA, ENDOD1, NFKB1, } \\
\text { BIRC3, TNFRSF10A, CASP10, PRKAR2B, IRAK3, TNFRSF10B, } \\
\text { PPP3CC, IL1B, PIK3R5, PIK3R3, IL1A }\end{array}$ & 3.51 & 2.1E-06 \\
\hline & hsa04621 & $\begin{array}{l}\text { NOD-like receptor signaling } \\
\text { pathway }\end{array}$ & $\begin{array}{l}\text { CXCL1, TNF, XIAP, IL8, RELA, CXCL2, NFKBIA, NFKB1, BIRC3, } \\
\text { NOD2, RIPK2, IL1B, TNFAIP3 }\end{array}$ & 3.20 & 5.4E-04 \\
\hline & hsa04060 & $\begin{array}{l}\text { Cytokine-cytokine receptor } \\
\text { interaction }\end{array}$ & $\begin{array}{l}\text { CXCL1, TNFRSF21, CCL3, TNF, CXCL5, CXCL3, CXCL2, } \\
\text { TNFSF15, CXCL6, IL7R, CCL4, TGFB2, LIF, CCL22, IL23A, CCL20, } \\
\text { CCL3L1, IL4R, TNFRSF18, IL15RA, IL1B, IL1A, BMP2, IL8, CD40, } \\
\text { IL11RA, TNFRSF10A, INHBA, ACVR2B, TNFRSF10B, VEGFA }\end{array}$ & 1.81 & 1.6E-03 \\
\hline & hsa04062 & $\begin{array}{l}\text { Chemokine signaling } \\
\text { pathway }\end{array}$ & $\begin{array}{l}\text { CXCL1, ADCY4, CCL3, LYN, CXCL5, IL8, HCK, CXCL3, RELA, } \\
\text { CXCL2, NFKBIA, ADRBK2, NFKB1, CXCL6, CCL4, CCL22, } \\
\text { CCL20, CCL3L1, GNG10, SOS2, PIK3R5, GNB4, PIK3R3, GNG7 }\end{array}$ & 1.96 & 2.2E-03 \\
\hline & hsa05222 & Small cell lung cancer & $\begin{array}{l}\text { E2F1, TRAF1, XIAP, PTGS2, RELA, TP53, ITGA2, NFKBIA, NFKB1, } \\
\text { BIRC3, LAMB3, PIK3R5, PIK3R3, TRAF3 }\end{array}$ & 2.55 & 2.8E-03 \\
\hline & hsa05200 & Pathways in cancer & $\begin{array}{l}\text { TRAF1, E2F1, BID, PTGS2, XIAP, STAT5A, MITF, NFKBIA, NFKB1, } \\
\text { NFKB2, TCF7L2, MMP1, TGFB2, LAMB3, SOS2, PIK3R5, CCNA1, } \\
\text { PIK3R3, FGF2, TRAF3, BMP2, IL8, VHL, RELA, TP53, ITGA2, } \\
\text { BIRC5, BIRC3, FZD4, DAPK3, CTNNA3, RAD51, SMO, ETS1, } \\
\text { VEGFA }\end{array}$ & 1.63 & 4.2E-03 \\
\hline & hsa04620 & $\begin{array}{l}\text { Toll-like receptor signaling } \\
\text { pathway }\end{array}$ & $\begin{array}{l}\text { CCL3, TNF, IL8, RELA, NFKBIA, NFKB1, CD40, CCL4, CD86, } \\
\text { MAP3K8, IL1B, PIK3R5, PIK3R3, CD14, TRAF3 }\end{array}$ & 2.27 & 5.5E-03 \\
\hline Leishmania-infected & hsa00230 & Purine metabolism & $\begin{array}{l}\text { ADCY4, ADSSL1, ADCY8, POLA1, PDE4C, PDE6G, POLE4, } \\
\text { PDE2A, ADCY9, RRM2, PKLR, GUCY1A2, ADCY10, PRPS1 }\end{array}$ & 2.40 & 5.1E-03 \\
\hline
\end{tabular}

A

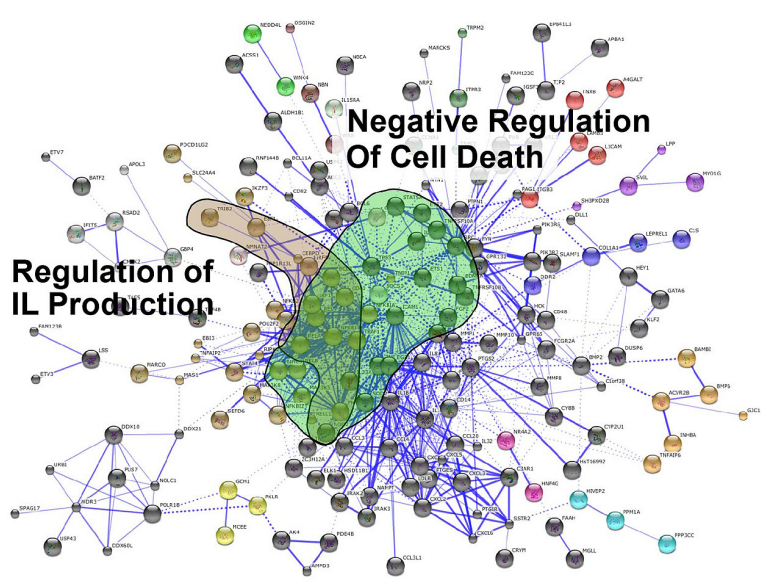

B

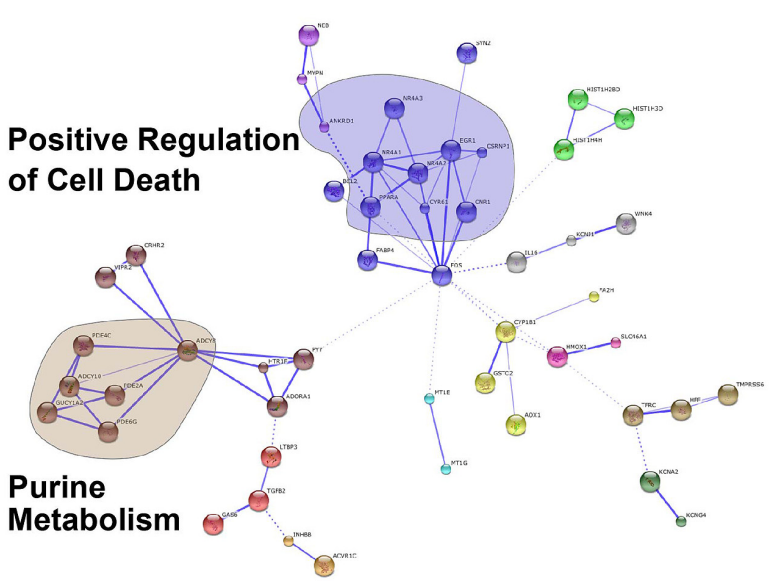

FIGURE 2 | Protein-protein interaction analysis. Protein-protein interaction networks of up-regulated genes in (A) C. burnetii-infected and (B) L. mexicanainfected THP1 cells visualized in STRING. Colors based on Markov Clustering with an inflation factor of 2. Highlighted clusters are labeled with their GO or KEGG categories.

regulated) were differentially expressed in C. burnetii infected THP-1 cells, whereas 698 genes (534 up-regulated and 164 down-regulated) were differentially expressed in L. mexicana infected cells (Figure 1, Supplementary Tables S1 and S2). Interestingly, the sets of genes affected by the two pathogens are very different. Of the 1755 total genes identified in this study, only 126 genes $(\sim 7 \%)$ are differentially expressed under both conditions, and no metabolic pathways were significantly enriched within this common set of genes (Figure 1, Supplementary Table S3). A previous study that compared THP-1 cell response to infections by Coxiella and Chlamydia trachomatis (an intracellular bacterium), reported an overlap of $\sim 25 \%$ of genes between the two infections (Ren et al., 2003). The low overlap between the host responses to Coxiella and Leishmania, and the higher magnitude of host response to C. burnetii than that to L. mexicana possibly reflects the more distant evolutionary relationship between the bacteria and the eukaryotic parasite compared to the two bacterial pathogens previously studied. Apoptosis and host cell immune response pathways were the most significantly enriched KEGG pathways in Coxiella infected cells (Table 1), as observed in previous microarray-based studies (Ren et al., 2003; Mahapatra et al., 
A

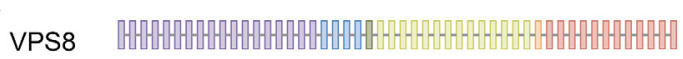

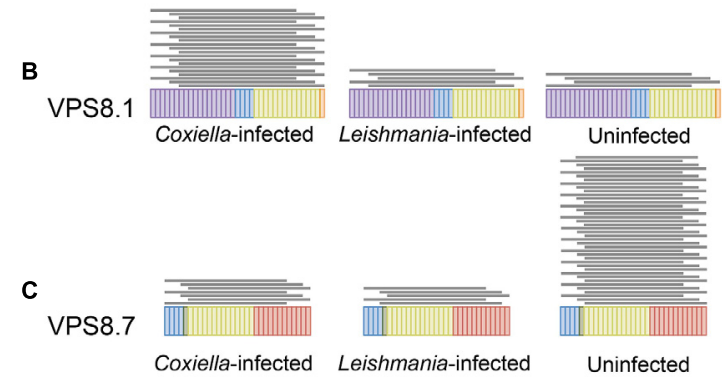

FIGURE 3 | Differential expression of mRNA isoforms. Differential isoform expression of VPS8 in C. burnetii infected, L. mexicana infected, and uninfected THP1 cells are shown as a representation of isoform analysis. (A) The full-length VPS8 gene is depicted with colored bars representing exons. Isoforms 1 (B) and 7 (C) of VPS8 that have significantly different expression in the three samples are shown. Each gray line above an isoform represents 10 mapped reads.

2010). Repression of host cell death by Coxiella has been reported previously (Lührmann and Roy, 2007; Voth et al., 2007), and is thought to promote intracellular growth of Coxiella within large PVs; conversely, induction of Toll-like Receptor signaling pathways and production of cytokines and chemokines participate in the host response to Coxiella infection.

In Leishmania-infected cells, purine metabolism was the only KEGG pathway that was significantly perturbed (Table 1). Leishmania is dependent on host for its purine supply (McConville et al., 2007), and three genes (ADSSL1, RRM2, PRPS1) involved in purine biosynthesis or salvage pathways were significantly overexpressed in infected THP-1 cells. Intriguingly, a majority of "purine metabolism" genes listed in Table 1 regulate the levels of intracellular second messengers cAMP and cGMP. Adenylate cyclases (ADCY4, ADCY8, ADCY9,
ADCY10) catalyze the formation of cAMP from ATP; guanylate cyclase (GUCY1A2) catalyzes the conversion of GTP to cGMP; phosphodiesterases (PDE4C, PDE6G, PDE2A) catalyze the hydrolysis of cAMP and/or cGMP. Previous studies have shown that Leishmania resists host antimicrobial activities by modulating several host signaling pathways, including $\mathrm{Ca}^{2+}$ and PKC-dependent pathways, JAK-STAT pathways, and MAP kinases (Olivier et al., 2005). Similarly, Leishmania could be subverting the host's cAMP and cGMP signaling pathways in order to suppress immune responses and to promote its intracellular growth.

A protein-protein interaction network analysis using the STRING database (Franceschini et al., 2013) confirmed that Coxiella infection induced the expression of genes involved in negative regulation of cell death (Figure 2A). In contrast, this analysis identified that genes involved in positive regulation of cell death were upregulated in Leishmania-infected cells (Figure 2B). We confirmed this trend by analyzing the expression of a subset of cell death-related genes using qPCR (Additional File Supplementary Figure S1). The induction of host cell death during later stages of infection probably aids in the cell-to-cell transfer of Leishmania amastigotes within membrane blebs, as shown recently (Real et al., 2014).

\section{Differential Expression of mRNA Isoforms in Infected and Uninfected Cells}

In human cells, alternate splicing of pre-mRNA can give rise to several isoforms of the mature mRNA, and proteins derived from them may have distinct cellular roles (Lareau et al., 2004). In addition to expanding the proteome, cells utilize alternate splicing as a regulatory tool. For example, a short splice variant of human tryptophan-tRNA synthase, but not the full length protein, regulates angiogenesis (Wakasugi et al., 2002). Isoform generation may also have a role in host cell response against infections. Different isoforms of p53 (encoded by TP53 gene) are involved in host defense against both bacterial (Helicobacter pylori) and viral (Influenza and Simian virus 40)

TABLE 2 | MicroRNAs (miRNAs) perturbed by Coxiella and Leishmania infections.

\begin{tabular}{|c|c|c|c|c|c|}
\hline Sample & miRNA & Fold change (log2) & $P$-value & Regulation & Process \\
\hline \multirow{5}{*}{ Coxiella-infected } & mir-181d-5p & 0.78 & $<0.001$ & Up & Anti-apoptotic ${ }^{b}$ \\
\hline & mir-362-5p & 0.89 & 0.015 & Up & Anti-apoptotic ${ }^{d}$ \\
\hline & mir-361-5p & 0.95 & 0.004 & Up & Anti-apoptotic ${ }^{e}$ \\
\hline & mir-194-2-5p & 1.05 & 0.024 & Up & Anti-apoptotic $^{f}$ \\
\hline & mir-28-5p & 1.35 & $<0.001$ & Up & Pro-apoptotic ${ }^{g}$ \\
\hline \multirow[t]{5}{*}{ Leishmania-infected } & mir-145-5p & -1.00 & 0.002 & Down & Pro-apoptotic ${ }^{h}$ \\
\hline & mir-221-5p & -0.62 & $<0.001$ & Down & Anti-apoptotici \\
\hline & mir- $15 b-5 p$ & 0.56 & 0.035 & Up & Pro-apoptotic ${ }^{j}$ \\
\hline & mir-29b-1-3p & 1.09 & 0.002 & Up & Pro-apoptotick \\
\hline & mir-29b-2-3p & 1.19 & $<0.001$ & Up & Pro-apoptotick \\
\hline
\end{tabular}

a Zhang et al. (2011), 'b Wang et al. (2010), ' Nakano et al. (2013), ' Xia et al. (2014), e'Wu et al. (2013), ' Zhang et al. (2014), g Almeida et al. (2012), ${ }^{\mathrm{h}}$ Davis-Dusenbery et al. (2011), ile Sage et al. (2007), i 'Cimmino et al. (2005), kGarzon et al. (2009). 
infections (Terrier et al., 2013). Similarly, Hepatitis C virus activates the immunologic isoform of nitric oxide synthase (NOS) gene, which induces NO production (Machida et al., 2004).

Transcriptome analysis (RNA-seq) is a powerful approach to identify differential isoform expression under different conditions at a genome-wide scale (Eswaran et al., 2013; Lo et al., 2014). We used RNA-seq to investigate whether infection by either Coxiella or Leishmania induced differential expression of human gene isoforms. We identified 689 isoforms from 626 genes that were differentially expressed in C. burnetii-infected cells, and 651 isoforms from 569 genes in Leishmania-infected cells, when compared to uninfected THP-1 cells (Figure 3, Supplementary Tables S4 and S5). As observed for full-length mRNAs, there was minimal overlap between the sets of genes with differential expression of isoforms under each infection condition (only 105 common genes). Additionally, no KEGG pathways were significantly enriched in either gene set, indicating that differential isoform expression is a cell-wide phenomenon. Cumulatively, our data revealed that in addition to differences that are apparent at the gene level, the mostly unexplored realm of isoform variation could contribute to host responses to infections.

\section{Coxiella and Leishmania Infections Perturb the Expression of Apoptosis-Related miRNAs}

Expression of various protein-coding genes in humans is regulated by miRNAs. These small non-coding RNAs regulate the expression of target genes by base-pairing with mRNAs, thereby either blocking translation or causing target degradation or destabilization (Fabian et al., 2010). They are involved in many, if not all, biological processes, including metabolic pathways, cell proliferation, and apoptosis. Recently, miRNAs have been shown to be an important part of host cell response to viral, bacterial, and parasitic infections (Lagos et al., 2010; Schnitger et al., 2011; Schulte et al., 2011). In addition, some viruses, including Herpes viruses and Hepatitis $\mathrm{C}$ virus, have the ability to interfere with the host miRNA network to promote viral growth (Jopling et al., 2005; Cullen, 2011). Recent studies also showed that eukaryotic intracellular pathogens such as Cryptosporidium parvum and $T$. gondii promote intracellular replication by altering host cell miRNA networks (Hakimi and Ménard, 2010; Zeiner et al., 2010). To identify miRNAs that are potentially perturbed by C. burnetii or L. mexicana infections, we sequenced and enumerated miRNAs expressed by uninfected, Coxiellainfected, and Leishmania-infected THP-1 cells. We identified 257 miRNAs that were expressed in THP-1 cells (Supplementary Table S6), which includes 50 of the 64 miRNAs reported by a recent study that examined miRNAs expressed in human macrophages in response to Leishmania major infection (Lemaire et al., 2013). Among the 257 miRNAs, seven were upregulated and one was down regulated in Coxiella-infected cells, and three were upregulated and two were down regulated in Leishmaniainfected cells (Table 2). Intriguingly, several of the differentially expressed miRNAs have been shown in previous studies to regulate host cell death: miR-145 modulates the expression of KLF4 (Davis-Dusenbery et al., 2011), a transcription factor for TP53, which regulates apoptosis (Rowland et al., 2005); miR-15b and miR-29b are known to be pro-apoptotic in leukemia cells (Cimmino et al., 2005; Garzon et al., 2009); miR-148a promotes apoptosis by targeting BCL2 in colorectal cancer cells (Zhang et al., 2011); miR-181d also targets BCL-2 and promotes apoptosis in glioma cells (Wang et al., 2012). These results complement gene expression data (Figure 2), and indicate that miRNAs may have important roles in inhibiting host cell death during Coxiella infection, and promoting host cell death during Leishmania infection.

\section{Conclusion}

The genome-wide gene, mRNA-isoform, and miRNA expression patterns were distinct between macrophages infected with either C. burnetii or L. mexicana, indicating that even though both pathogens have converged on a similar intracellular niche, they utilize distinct programs to generate and maintain their respective PVs.

\section{Author Contributions}

RR, EC, and SL designed the study. RV, FK, and EC carried out the experiments. JM and RR analyzed the data and drafted the manuscript. All authors read and approved the final manuscript.

\section{Acknowledgments}

We thank Christine Sislak and Abraham Moses for technical assistance. This work was supported in part by grants from American Heart Association and Medical Research Foundation (to RR), National Institutes of Health Grant AI44138 (to SL) and National Institutes of Health Grant AI088275 (to EC).

\section{Supplementary Material}

The Supplementary Material for this article can be found online at: http://journal.frontiersin.org/article/10.3389/fmicb. 2015.00794

FIGURE S1 | Validation by qPCR of expression levels estimated by RNA-seq. Fold difference values calculated by GPCR correlated well with fold difference values calculated by RNA-seq.

TABLE S1 | Differentially expressed genes in Coxiella-infected vs. uninfected THP-1 cells.

TABLE S2 | Differentially expressed genes in Leishmania-infected vs. uninfected THP-1 cells.

TABLE S3 | Differentially expressed genes that overlap between Coxiella-infected vs. uninfected and Leishmania-infected vs. uninfected THP-1 cells.

TABLE S4 | Differentially expressed mRNA isoforms in Coxiella-infected vs. uninfected THP-1 cells.

TABLE S5 | Differentially expressed mRNA isoforms in Leishmaniainfected vs. uninfected THP-1 cells.

TABLE S6 | All miRNAs detected in this study. 


\section{References}

Alix, E., Mukherjee, S., and Roy, C. R. (2011). Subversion of membrane transport pathways by vacuolar pathogens. J. Cell Biol. 195, 943-952. doi: $10.1083 /$ jcb. 201105019

Almeida, M., Nicoloso, M., Zeng, L., and Ivan, C. (2012). Strand-specific miR-28-5p and $\mathrm{miR}-28-3 \mathrm{p}$ have distinct effects in colorectal cancer cells. Gastroenterology 142, 886-896. doi: 10.1053/j.gastro.2011.12.047

Beare, P. A., Gilk, S. D., Larson, C. L., Hill, J., Stead, C. M., Omsland, A., et al. (2011). Dot/Icm type IVB secretion system requirements for Coxiella burnetii growth in human macrophages. MBio 2, 1-10. doi: 10.1128/mBio.00175-11

Bolger, A. M., Lohse, M., and Usadel, B. (2014). Trimmomatic: a flexible trimmer for Illumina sequence data. Bioinformatics 30, 2114-2120. doi: 10.1093/bioinformatics/btu170

Brohée, S., and van Helden, J. (2006). Evaluation of clustering algorithms for protein-protein interaction networks. BMC Bioinform. 7:488. doi: 10.1186/1471-2105-7-488

Cimmino, A., Calin, G. A., Fabbri, M., Iorio, M. V., Ferracin, M., Shimizu, M. et al. (2005). miR-15 and miR-16 induce apoptosis by targeting BCL2. Proc. Natl. Acad. Sci. U.S.A. 102, 13944-13949. doi: 10.1073/pnas.0506654102

Contreras, I., Estrada, J. A., Guak, H., Martel, C., Borjian, A., Ralph, B., et al. (2014). Impact of Leishmania mexicana infection on dendritic cell signaling and functions. PLoS Negl. Trop. Dis. 8:e3202. doi: 10.1371/journal.pntd.0003202

Cullen, B. R. (2011). Viruses and microRNAs: RISCy interactions with serious consequences. Genes Dev. 25, 1881-1894. doi: 10.1101/gad.17352611

Davis-Dusenbery, B. N., Chan, M. C., Reno, K. E., Weisman, A. S., Layne, M. D., Lagna, G., et al. (2011). Down-regulation of Kruppel-like factor-4 (KLF4) by microRNA-143/145 is critical for modulation of vascular smooth muscle cell phenotype by transforming growth factor-beta and bone morphogenetic protein 4. J. Biol. Chem. 286, 28097-28110. doi: 10.1074/jbc.M111.236950

De Muylder, G., Ang, K. K. H., Chen, S., Arkin, M. R., Engel, J. C., and McKerrow, J. H. (2011). A screen against Leishmania intracellular amastigotes: comparison to a promastigote screen and identification of a host cell-specific hit. PLoS Negl. Trop. Dis. 5:e1253. doi: 10.1371/journal.pntd.0001253

Eswaran, J., Horvath, A., Godbole, S., Reddy, S. D., Mudvari, P., Ohshiro, K., et al. (2013). RNA sequencing of cancer reveals novel splicing alterations. Sci. Rep. 3:1689. doi: 10.1038/srep01689

Fabian, M. R., Sonenberg, N., and Filipowicz, W. (2010). Regulation of mRNA translation and stability by microRNAs. Annu. Rev. Biochem. 79, 351-379. doi: 10.1146/annurev-biochem-060308-103103

Flannagan, R. S., Cosío, G., and Grinstein, S. (2009). Antimicrobial mechanisms of phagocytes and bacterial evasion strategies. Nat. Rev. Microbiol. 7, 355-366. doi: $10.1038 /$ nrmicro2128

Franceschini, A., Szklarczyk, D., Frankild, S., Kuhn, M., Simonovic, M., Roth, A., et al. (2013). STRING v9.1: protein-protein interaction networks, with increased coverage and integration. Nucl. Acids Res. 41, D808-D815. doi: 10.1093/nar/gks1094

Garzon, R., Heaphy, C. E. A., Havelange, V., Fabbri, M., Volinia, S., Tsao, T., et al. (2009). MicroRNA 29b functions in acute myeloid leukemia. Blood 114, 5331-5342. doi: 10.1182/blood-2009-03-211938

Hakimi, M. A., and Ménard, R. (2010). Do apicomplexan parasites hijack the host cell microRNA pathway for their intracellular development? Biol. Rep. 2, 16-18. doi: 10.3410/B2-42

Herwaldt, B. L. (1999). Leishmaniasis. Lancet 354, 1191-1199. doi: 10.1016/S01406736(98)10178-10172

Huang, D. W., Sherman, B., and Lempicki, R. (2009a). Systematic and integrative analysis of large gene lists using DAVID bioinformatics resources. Nat. Protoc. 4, 44-57. doi: 10.1038/nprot.2008.211

Huang, D. W., Sherman, B. T., and Lempicki, R. A. (2009b). Bioinformatics enrichment tools: paths toward the comprehensive functional analysis of large gene lists. Nucl. Acids Res. 37, 1-13. doi: 10.1093/nar/gkn923

Jopling, C., Yi, M., Lancaster, A., Lemon, S., and Samow, P. (2005). Modulation of hepatitis C virus RNA abundance by a liver-specific MicroRNA. Science 309, 1577-1581. doi: 10.1126/science.1113329

Kim, D., Pertea, G., Trapnell, C., Pimentel, H., Kelley, R., and Salzberg, S. L. (2013). TopHat2: accurate alignment of transcriptomes in the presence of insertions, deletions and gene fusions. Genome Biol. 14:R36. doi: 10.1186/gb-2013-14$4-\mathrm{r} 36$
Kinchen, J. M., and Ravichandran, K. S. (2008). Phagosome maturation: going through the acid test. Nat. Rev. Mol. Cell Biol. 9, 781-795. doi: 10.1038/ nrm 2515

Kozomara, A., and Griffiths-Jones, S. (2014). miRBase: annotating high confidence microRNAs using deep sequencing data. Nucl. Acids Res. 42, D68-D73. doi: 10.1093/nar/gkt1181

Lagos, D., Pollara, G., Henderson, S., Gratrix, F., Fabani, M., Milne, R. S. B., et al. (2010). miR-132 regulates antiviral innate immunity through suppression of the p300 transcriptional co-activator. Nat. Cell Biol. 12, 513-519. doi: $10.1038 /$ ncb2054

Lareau, L. F., Green, R. E., Bhatnagar, R. S., and Brenner, S. E. (2004). The evolving roles of alternative splicing. Curr. Opin. Struct. Biol. 14, 273-282. doi: 10.1016/j.sbi.2004.05.002

Lemaire, J., Mkannez, G., Guerfali, F. Z., Gustin, C., Attia, H., Sghaier, R. M., et al. (2013). MicroRNA expression profile in human macrophages in response to Leishmania major infection. PLoS Negl. Trop. Dis. 7:e2478. doi: 10.1371/journal.pntd.0002478

Leng, N., Dawson, J. A., Thomson, J. A., Ruotti, V., Rissman, A. I., Smits, B. M. G., et al. (2013). EBSeq: an empirical bayes hierarchical model for inference in RNA-seq experiments. Bioinformatics 29, 1035-1043. doi: 10.1093/bioinformatics/btt087

le Sage, C., Nagel, R., Egan, D. A., Schrier, M., Mesman, E., Mangiola, A., et al. (2007). Regulation of the p27 (Kip1) tumor suppressor by miR-221 and miR-222 promotes cancer cell proliferation. EMBO J. 26, 3699-3708. doi: 10.1038/sj.emboj.7601790

Li, H., and Durbin, R. (2010). Fast and accurate long-read alignment with Burrows-Wheeler transform. Bioinformatics 26, 589-595. doi: 10.1093/bioinformatics/btp698

Lo, W. S., Gardiner, E., Xu, Z., Lau, C. F., Wang, F., Zhou, J. J., et al. (2014). Human tRNA synthetase catalytic nulls with diverse functions. Science 345, 328-332. doi: 10.1126/science.1252943

Lührmann, A., and Roy, C. R. (2007). Coxiella burnetii inhibits activation of host cell apoptosis through a mechanism that involves preventing cytochrome c release from mitochondria. Infect. Immun. 75, 5282-5289. doi: 10.1128/IAI.00863-7

Machida, K., Cheng, K. T. H., Sung, V. M. H., Lee, K. J., Levine, A. M., and Lai, M. M. C. (2004). Hepatitis C virus infection activates the immunologic (type II) isoform of nitric oxide synthase and thereby enhances DNA damage and mutations of cellular genes. J. Virol. 78, 8835-8843. doi: 10.1128/JVI.78.16.8835-8843.2004

Mahapatra, S., Ayoubi, P., and Shaw, E. I. (2010). Coxiella burnetii nine mile II proteins modulate gene expression of monocytic host cells during infection. BMC Microbiol. 10:244. doi: 10.1186/1471-2180-10-244

McConville, M. J., de Souza, D., Saunders, E., Likic, V. A., and Naderer, T. (2007). Living in a phagolysosome; metabolism of Leishmania amastigotes. Trends Parasitol. 23, 368-375. doi: 10.1016/j.pt.2007.06.009

Nakano, H., Yamada, Y., Miyazawa, T., and Yoshida, T. (2013). Gain-offunction microRNA screens identify miR-193a regulating proliferation and apoptosis in epithelial ovarian cancer cells. Int. J. Oncol. 42, 1875-1882. doi: 10.3892/ijo.2013.1896

Newton, H. J., and Roy, C. R. (2011). The Coxiella burnetii Dot/Icm system creates a comfortable home through lysosomal renovation. MBio 2:e00226-11. doi: 10.1128/mBio.00226-11

Olivier, M., Gregory, D. J., and Forget, G. (2005). Subversion mechanisms by which Leishmania parasites can escape the host immune response: a signaling point of view. Clin. Microbiol. Rev. 18, 293-305. doi: 10.1128/CMR.18.2.293-30 5.2005

Rabhi, I., Rabhi, S., Ben-Othman, R., Aniba, M. R., Trentin, B., Piquemal, D., et al. (2013). Comparative analysis of resistant and susceptible macrophage gene expression response to Leishmania major parasite. BMC Genomics 14:723. doi: 10.1186/1471-2164-14-723

Rabhi, I., Rabhi, S., Ben-Othman, R., Rasche, A., Daskalaki, A., Trentin, B., et al. (2012). Transcriptomic signature of Leishmania infected mice macrophages: a metabolic point of view. PLoS Negl. Trop. Dis. 6:e1763. doi: 10.1371/journal.pntd.0001763

Rabinovitch, M., and Veras, P. S. T. (1996). Cohabitation of Leishmania amazonensis and Coxiella burnetii. Trends Microbiol. 4, 158-161. doi: 10.1016/0966-842X(96)10027-10025 
Raghavan, R., Sloan, D. B., and Ochman, H. (2012). Antisense transcription is pervasive but rarely conserved in enteric bacteria. MBio 3:e00156-12. doi: 10.1128/mBio.00156-112

Real, F., Florentino, P. T. V., Reis, L. C., Ramos-Sanchez, E. M., Veras, P. S. T., Goto, H., et al. (2014). Cell-to-cell transfer of Leishmania amazonensis amastigotes is mediated by immunomodulatory LAMP-rich parasitophorous extrusions. Cell. Microbiol. 16, 1549-1564. doi: 10.1111/cmi.12311

Real, F., Mortara, R. A., and Rabinovitch, M. (2010). Fusion between Leishmania amazonensis and Leishmania major parasitophorous vacuoles: live imaging of coinfected macrophages. PLoS Negl. Trop. Dis. 4:e905. doi: 10.1371/journal.pntd.0000905

Ren, Q., Robertson, S., Howe, D., Barrows, L., and Heinzen, R. (2003). Comparative DNA microarray analysis of host cell transcriptional responses to infection by Coxiella burnetii or Chlamydia trachomatis. Ann. N. Y. Acad. Sci. 990, 701-713. doi: 10.1111/j.1749-6632.2003.tb07447.x

Rowland, B. D., Bernards, R., and Peeper, D. S. (2005). The KLF4 tumour suppressor is a transcriptional repressor of p53 that acts as a context-dependent oncogene. Nat. Cell Biol. 7, 1074-1082. doi: 10.1038/ncb1314

Schnitger, A. K. D., Machova, A., Mueller, R. U., Androulidaki, A., Schermer, B., Pasparakis, M., et al. (2011). Listeria monocytogenes infection in macrophages induces vacuolar-dependent host miRNA response. PLOS ONE 6:e27435. doi: 10.1371/journal.pone.0027435

Schulte, L. N., Eulalio, A., Mollenkopf, H. J., Reinhardt, R., and Vogel, J. (2011). Analysis of the host microRNA response to Salmonella uncovers the control of major cytokines by the let-7 family. EMBO J. 30, 1977-1989. doi: 10.1038/emboj.2011.94

Swanson, M. S., and Fernandez-Moreia, E. (2002). A microbial strategy to multiply in macrophages: the pregnant pause. Traffic 3, 170-177. doi: $10.1034 /$ j.16000854.2002.030302.x

Terrier, O., Bourdon, J. C., and Rosa-Calatrava, M. (2013). P53 protein isoforms: key regulators in the front line of pathogen infections? PLoS Pathog. 9:e1003246. doi: 10.1371/journal.ppat.1003246

van Schaik, E. J., Chen, C., Mertens, K., Weber, M. M., and Samuel, J. E. (2013). Molecular pathogenesis of the obligate intracellular bacterium Coxiella burnetii. Nat. Rev. Microbiol. 11, 561-573. doi: 10.1038/nrmicro3049

Veras, P. S. T., Moulia, C., Dauguet, C., Tunis, C. T., Thibon, M., and Rabinovitch, M. (1995). Entry and survival of Leishmania amazonensis amastigotes within phagolysosome-like vacuoles that shelter Coxiella burnetii in Chinese hamster ovary cells. Infect. Immun. 63, 3502-3506.

Voth, D. E., and Heinzen, R. A. (2007). Lounging in a lysosome: the intracellular lifestyle of Coxiella burnetii. Cell. Microbiol. 9, 829-840. doi: 10.1111/j.14625822.2007.00901.x
Voth, D. E., Howe, D., and Heinzen, R. A. (2007). Coxiella burnetii inhibits apoptosis in human THP-1 cells and monkey primary alveolar macrophages. Infect. Immun. 75, 4263-4271. doi: 10.1128/IAI.00594-597

Wakasugi, K., Slike, B. M., Hood, J., Otani, A., Ewalt, K. L., Friedlander, M., et al. (2002). A human aminoacyl-tRNA synthetase as a regulator of angiogenesis. Proc. Natl. Acad. Sci. U.S.A. 99, 173-177. doi: 10.1073/pnas.012602099

Wang, B., Hsu, S. H., Majumder, S., Kutay, H., Huang, W., Jacob, S. T., et al. (2010). TGFbeta-mediated upregulation of hepatic miR-181b promotes hepatocarcinogenesis by targeting TIMP3. Oncogene 29, 1787-1797. doi: 10.1038/onc.2009.468

Wang, X. F., Shi, Z. M., Wang, X. R., Cao, L., Wang, Y. Y., Zhang, J. X., et al. (2012). MiR-181d acts as a tumor suppressor in glioma by targeting K-ras and Bcl-2. J. Cancer Res. Clin. Oncol. 138, 573-584. doi: 10.1007/s00432-011-1114-X

Wu, X., Xi, X., Yan, Q., Zhang, Z., Cai, B., Lu, W., et al. (2013). MicroRNA361-5p facilitates cervical cancer progression through mediation of epithelialto-mesenchymal transition. Med. Oncol. 30:751. doi: 10.1007/s12032-0130751-0

Xia, J., Chen, L., Jian, W., Wang, K., Yang, Y., He, W., et al. (2014). MicroRNA-362 induces cell proliferation and apoptosis resistance in gastric cancer by activation of NF- $\kappa$ B signaling. J. Trans. Med. 12, 33. doi: 10.1186/1479-5876-12-33

Zeiner, G. M., Norman, K. L., Thomson, J. M., Hammond, S. M., and Boothroyd, J. C. (2010). Toxoplasma gondii infection specifically increases the levels of key host microRNAs. PLoS ONE 5:e8742. doi: 10.1371/journal.pone.0008742

Zhang, H., Li, Y., Huang, Q., Ren, X., Hu, H., Sheng, H., et al. (2011). MiR-148a promotes apoptosis by targeting Bcl-2 in colorectal cancer. Cell Death Diff. 18, 1702-1710. doi: 10.1038/cdd.2011.28

Zhang, J., Zhao, C. Y., Zhang, S. H., Yu, D. H., Chen, Y., Liu, Q. H., et al. (2014). Upregulation of miR-194 contributes to tumor growth and progression in pancreatic ductal adenocarcinoma. Oncol. Rep. 31, 1157-1164. doi: $10.3892 /$ or. 2013.2960

Conflict of Interest Statement: The authors declare that the research was conducted in the absence of any commercial or financial relationships that could be construed as a potential conflict of interest.

Copyright (C) 2015 Millar, Valdés, Kacharia, Landfear, Cambronne and Raghavan. This is an open-access article distributed under the terms of the Creative Commons Attribution License (CC BY). The use, distribution or reproduction in other forums is permitted, provided the original author(s) or licensor are credited and that the original publication in this journal is cited, in accordance with accepted academic practice. No use, distribution or reproduction is permitted which does not comply with these terms. 